\title{
Asi
}

\section{La política de inserción por la economía en Francia}

\section{EGUZKI URTEAGA}

DEPARTAMENTO DE SOCIOLOGÍA

UNIVERSIDAD DEL PAÍS VASCO

eguzki.urteaga@ehu.es

Resumen: En las últimas tres décadas, Francia ha reconocido un derecho a la inserción a las personas que se hallaban en una situación de vulnerabilidad social, a pesar de que puedan distinguirse varios periodos. La aprobación de la Renta Mínima de Inserción (RMI) y la creación de estructuras especializadas en la inserción por la economía, especialmente en dirección de los jóvenes, han significado un cambio de filosofía, puesto que se abandona el derecho a la asistencia para acceder al derecho a la inserción. Gracias a unos cambios legislativos, con la aprobación de la ley de lucha contra las exclusiones y de la ley de cohesión social, esta política ha dado lugar, por una parte, a la creación de estructuras adecuadas, sinónimas de empresas de inserción, de asociaciones intermedias, de entidades de inserción o de agrupaciones de empleadores, y, por otra parte, a la aprobación de unas medidas individuales que se parecen a unos con- 
tratos subvencionados. La inserción por la actividad económica pretende favorecer la reinserción laboral de unos colectivos marginados lo que supone unas condiciones previas en materia de salud, de habilidades sociales y de capacidades cognitivas.

Palabras clave: política social, inserción, actividad económica, Francia.

\section{Reinsertion through economy policy in France}

Abstract: In the last three decades, France has recognized a right to the insertion to the persons who were situated in a situation of social vulnerability, in spite of the fact that several periods could be distinguished. The approval of the Minimal Revenue of Insertion (RMI) and the creation of structures specialized in the insertion by the economy, specially in direction of the young persons, have meant a change of philosophy, since the right of the assistance to the right of the insertion. Thanks to a few legislative changes, with the approval of the law of fight against the exclusions and the law of social cohesion, this policy has given place, on one hand, to the creation of suitable structures, synonymous of companies of insertion, of intermediate associations, of entities of insertion or of employers' groups, and, on the other hand, the approval of a few individual measures that are feigned for helped contracts. The insertion for the economic activity tries to favor the labou rehabilitation of a few isolated groups, which supposes a few previous conditions as for health, social skills and cognitive capacities.

Keywords: social politics, insertion, economic activity, France.

\section{La política de inserción por la economía en Francia}

Eguzki Urteaga

\section{Introducción}

A lo largo de los últimos treinta años, Francia ha favorecido el desarrollo de un derecho a la inserción, especialmente vía la Renta Mínima de Inserción (RMI) ${ }^{1}$ y las estructuras de inserción por la economía, pasando así de un derecho a la asistencia a un derecho a la inserción. Esta política se ha traducido a la vez por la creación de estructuras especializadas (empresas de inserción, asociaciones de inserción, agrupaciones de empleadores para la inserción y formación) y unas medidas individuales bajo la forma de contratos subvencionados (Contratos Empleo Solidaridad, Contratos Iniciativas Empleo, Empleos Jóvenes, Contratos Empleo Consolidado). La inserción a través de la economía, que se ha convertido en la inserción por la actividad económica, es uno de los apartados de la economía social y solidaria. Reúne a un conjunto de entidades y de dispositivos destinados a favorecer la reinserción laboral de unos colectivos fragilizados o marginados para los cuales las perspectivas de acceso al empleo suponen unas condiciones previas en materia de salud y de habilidad social para que puedan conservar un trabajo de manera duradera.

1 La RMI es una prestación social concedida desde el 1 de diciembre de 1988 por las Cajas de Ayuda Familiar y por la Mutualidad Social Agrícola a las personas cuyos recursos son inferiores a una cuantía fijada por el Gobierno. En marzo de 2007, 1229754 personas recibían esta prestación, de las cuales la mayoría vivían solas. La RMI ha sido sustituida por la Renta de Solidaridad Activa (RSA) el 1 de junio de 2009 


\section{De los años 1950 a los años 1970}

Al inicio de los años 1950, las primeras comunidades de Emmaüs inventan el concepto de activación de las personas como medio de lucha contra la exclusión. En 1974, la ley, que reconoce los Centros de alojamiento y de reinserción social $(\mathrm{CARS})^{2}$, pone el énfasis sobre las actividades de inserción. Asimismo, la circular 44, publicada el 10 de septiembre de 1979 por el ministerio de Sanidad y de Seguridad social, constituye el marco legal de la inserción por la economía a través de la creación de unos Centros de adaptación a la vida activa (CAVA) ${ }^{3}$ Están destinados a facilitar la reinserción social de las personas amenazadas de inadaptación y se organizan en tres tipos de estructuras: los talleres de aproximación al trabajo y al esfuerzo los centros de día para las personas cuyo retorno al empleo no protegido parece difícilmente concebible; los servicios de colocación que permiten trabajar fuera, bajo el control del centro, y gestionados principalmente por los CARS. No obstante, conocen un éxito limitado y se mezclan progresivamente a los demás dispositivos generales de inserción.

En los años 1970, los jóvenes son los primeros afectados por el incremento del desempleo. En 1975, las primeras medidas de empleo-formación son puestas en marcha, pero tienen una duración limitada puesto que se interrumpen a partir del mes de junio de 1976. Poco después, en 1977, la idea es retomada y encarnada por los tres pactos para el empleo conocidos como las "prácticas Barre", del nombre del Primer ministro de la época. A término de estas prácticas, cerca de 500000 jóvenes son contratados gracias a una exoneración de cargas sociales para las

${ }^{2}$ Las CARS tienen como objetivo: la acogida, el alojamiento, el acompañamiento y la inserción laboral de las personas en búsqueda de alojamiento o de vivienda con el fin de permitirles acceder a una autonomía personal y social. Teniendo en cuenta sus misiones, los CARS intervienen en diferentes ámbitos de decisión a propósito de las políticas sociales locales. Las CARS forman parte igualmente del "dispositivo invernal de acogida de urgencia", es decir igualmente del "dispositivo invernal de acogida de urgencia", es decir c capacidades de acogida de urgencia aumentan durante este periodo.

${ }^{3}$ Los CAVA realizan, tras la firma de un contrato, unas obras que no obedecen a las reglas del mercado y son el objeto de un convenio específico con e ministerio de Sanidad. No se trata de unas estructuras regidas por el derecho laboral y las personas que trabajan en estos talleres reciben una remuneración que varía entre el $30 \%$ y el $80 \%$ del salario mínimo interprofesional. empresas. Son creados unos espacios de trabajo de tipo "talleres de producción", llevados a cabo fundamentalmente por el sector de la prevención especializada y, por lo tanto, por los educadores. La circular 44 pretende reconocer estas iniciativas y ofrecer un marco jurídico a estas empresas de un nuevo tipo.

\section{Los años 1980}

En 1981, el gobierno socialista, recién llagado al poder, convierte a la inserción socio-profesional de los jóvenes en una preocupación central, ya que, en esta época, el $45 \%$ de los desempleados tiene menos de 25 años y los jóvenes que se hallan en el paro representan a 900000 personas. En este contexto, Bernard Schwartz entrega un informe al gobierno titulado "la inserción profesional y social de los jóvenes en dificultad". Elaborado a partir de las contribuciones y experiencias de más de 400 profesionales de la inserción, pone en evidencia cinco principios fundamentales para llevar a cabo una política eficaz a favor de la inserción de los jóvenes: 1) la proximidad o la capacidad de superación de los jóvenes, 2) la toma en consideración integral de sus dificultades, 3) la participación activa de los jóvenes, 4) la discriminación positiva a favor de los que se encuentran en dificultad y 5) la innovación para inventar nuevas respuestas.

Se crean las Misiones jóvenes el 26 de marzo de 1982. Se destinan a la orientación y al apoyo personalizado de los jóvenes de entre 16 y 25 años que se hallan en dificultad de inserción social y profesional. Concebidas como unos espacios de cooperación de proximidad, no deben crear un nuevo servicio sino favorecer la puesta en común de las energías locales. Están fundamentadas sobre un principio de colaboración institucional y de corresponsabilidad con una fuerte implicación de los ayuntamientos. Son creadas, poco a poco, más de 300 Misiones locales y otras tantas Permanencias de acogida, de información y de orientación (PAIO) ${ }^{4}$.

${ }^{4}$ Las PAIO asesoran y orientan a los jóvenes de entre 16 y 25 años en su trayectoria profesional así como en su vida diaria. Estas estructuras asociativas se encargan de acoger, informar, orientar y ayudar a los jóvenes en sus gestiones para conseguir su inserción profesional y social. Dan unas respuestas ade- 
La alternancia, qua une la formación académica y la experiencia profesional, debe ayudar a los jóvenes menos formados a encontrar un empleo. La dimensión de la inserción social es tomada en consideración como un aspecto inseparable de la inserción profesional, de manera que la inserción económica se convierte en la palabra clave de la ley del 19 de diciembre de 1989. La creación de los "Espacios jóvenes", en cooperación con los Consejos regionales y la ANPE (Agencia Nacional Para el Empleo $)^{5}$, marca esta evolución en torno a las acciones de "formación-empleo". Las nociones de territorio y de red local de inserción constituyen las bases de las nuevas estrategias. La búsqueda de vínculos crecientes con las empresas, la creación de clubes de patrocinadores y la contratación de antiguos empresarios como directores de las Misiones locales constituyen la prueba de ello.

La inserción social es confiada poco a poco a los actores tradicionales de la educación especializada, ya que los educadores de prevención especializada o de protección judicial de la juventud se encargan de implementar, en relación con los dispositivos existentes, unas acciones de inserción social. Una circular de 1983 sobre la política departamental de la educación vigilada presenta la inserción social y profesional como una prioridad. Entre la preparación de los jóvenes a una formación y una inserción profesional o la puesta en marcha de acciones humanitarias, deportivas, culturales, que resultan de la idea de un imposible acceso de estos jóvenes al empleo, los puntos de vista oscilan y se suceden. Encargados de movilizar a los jóvenes, los trabajadores sociales se enfrentan a la pérdida de esperanza provocada por los dispositivos sucesivos, que conducen los jóvenes de un cursillo a otro, sin disponer jamás de un verdadero empleo.

cuadas al conjunto de las dificultades a las que se enfrentan (vivienda, salud etc.) concediendo la prioridad al empleo y a la formación. Aseguran un seguimiento individualizado de los usuarios.

${ }^{5}$ Equivalente del INEM en Francia.

\section{La creación de las estructuras de inserción por la economía}

Tras numerosas iniciativas concretas, un programa experimental de "apoyo a las empresas intermedias" es elaborado y posteriormente aprobado a través de una circular del 24 de abril de 1985. Inicialmente destinado a los jóvenes de entre 16 y 25 años, es extendido en julio a los más de 26 años. Progresivamente, se crean unas empresas de inserción, a menudo bajo la egida de asociaciones existentes, pero el programa es suprimido por circular el 26 de septiembre de 1986. Puesto que la experiencia sólo ha afectado a un número limitado de jóvenes, el gobierno desea poner en marcha un programa de mayor envergadura. Bajo la presión de la Coordinación de las asociaciones de ayuda a los desempleados por el empleo (COORACE), aparecen las asociaciones intermedias que son instauradas por la ley del 27 de enero de 1987 y el decreto del 30 de abril de 1987. No afectan únicamente a los jóvenes sino también a cualquier persona que se encuentra en el desempleo. Contratan a personas en dificultad y las ponen a disposición de las empresas y de los ciudadanos.

El programa de las empresas intermedias es restablecido por una circular del 20 de abril de 1988. Desde entonces, estas empresas deben acoger en prioridad a personas en situación de marginación (toxicómanos, antiguos presos recién salidos de la cárcel o antiguos enfermos mentales que acaban de abandonar los hospitales psiquiátricos), tanto jóvenes como adultos. En 1989, su misión se extiende al conjunto de las personas en dificultad y en paro de larga duración. Se han reagrupado en 1988 en el comité nacional de las empresas de inserción. La puesta en marcha de las empresas de inserción abre un periodo de incertidumbre para las asociaciones intermedias que están consideradas como unas estructuras competidoras. Finalmente, la inserción a través de la economía y la diversidad de las estructuras son reconocidas por la ley del 3 de enero de 1991 que crea el Consejo nacional de la inserción por la actividad económica $(\mathrm{CNIAE})^{6}$.

${ }^{6}$ El CNIAE depende del Primer Ministro, y tanto la delegación general al empleo y a la formación profesional (DGEFP) como la dirección general de la acción social (DGAS) asumen la vicepresidencia. En respuesta a la solicitud del gobierno, la CNIAE asume tres misiones principales: 1) jugar un papel de ase- 
Un tercer actor aparece en la mitad de los años 1980: las corporaciones públicas de barrio a partir de la experiencia de la corporación pública de Roubaix. Situada en una posición intermedia entre la inserción económica y el desarrollo local, tienen como objetivo movilizar a los habitantes alrededor del mantenimiento de su entorno urbano y contribuir a la animación de barrio y a una misión social de inserción. En 1991, elaboran la Carta nacional de las corporaciones públicas de barrio y presentan el label "corporación pública de los barrios". Por último en 1993, los planes locales de inserción económica (PLIE) ${ }^{7}$ formalizan la cooperación local para construir unas trayectorias de inserción en función de unos objetivos cuantitativos. Completan los programas departamentales de inserción (PDI) ${ }^{8}$, que conciernen en primer lugar a los beneficiarios de la RMI.

La política soacial francesa resulta de una concepción que considera a los desempleados como a unas víctimas de los disfuncionamientos socioeconómicos. En esta perspectiva, se pone el énfasis sobre unas trayectorias de inserción que permiten a los individuos superar poco a poco sus hándicap sociales

soramiento y de vigilancia sobre las políticas de empleo y de inserción de las personas alejadas duraderamente del mercado laboral, 2) organizar la concertación entre los actores para desarrollar y consolidar el sector de la inserción po la actividad económica, especialmente favoreciendo la investigación, el desarrollo y difundiendo las experiencias y los métodos, y 3) representar la IAE en el Consejo nacional de las políticas de lucha contra la pobreza y la exclusión, en el Consejo superior del trabajo social, en el Consejo superior de la economía Cocial en el Obser dorio economico de la compra social y en los diferentes en los diferentes

Los PLIE resultan de las leyes y circulares que han tenido como objetivo la lucha contra la exclusión. Constituyen unos programas de puesta en coherencia de unas intervenciones públicas a nivel local con el fin de favorecer la reinserción profesional duradera de las personas en dificultad.

${ }^{8}$ Los PDI tratan el conjunto de los aspectos de la inserción: empleo, formación, vivienda, sanidad, acción social. Sus objetivos son, por una parte, satisfacer las necesidades a partir de la evaluación de las acciones existentes, y, por otra parte fijar los objetivos y los marcos de acción, identificar los medios correspondientes para multiplicar las iniciativas locales. Su actividad consiste fundapor 1) evaluar las necesidades y definir los objetivos en cada mentar en ámbito, 2) identificar las medidas existentes y asegurar el desarrollo de las nuevas acciones de inserción, 3) evaluar las necesidades de formación de los profesionales y de los voluntarios concernidos, 4) definir las condiciones de una coordinación entre los diferentes actores y una armonización de todas las medidas y 5) ampliarlas al conjunto de la lucha contra la pobreza y la exclusión. profesionales y familiares, y que limitan sus posibilidades de acceso al mercado laboral. En los países anglosajones, prevalece una concepción opuesta según la cual los excluidos son responsables de su situación. En esta óptica, la concesión de ayudas está sometida a una obligación de trabajo o de formación, de manera que la integración social se basa exclusivamente en el trabajo y se insiste en las obligaciones del individuo.

La vuelta al trabajo y el acompañamiento social son las dos caras de una misma misión que algunos prefieren distinguir en función de las competencias consideradas como específicas. Se trata entonces de evitar la confusión entre la producción y el apoyo social, y de promover una lógica de red que permita una mayor autonomía de la persona. La profesionalización del acompañamiento social conduce, en el marco de la inserción por la economía, a unas situaciones cada vez más complejas. La noción de contrato ha sustituido poco a poco la automaticidad de las prestaciones sociales. Los contratos de inserción puestos en marcha masivamente en el marco de la RMI a partir de los años 1990 traducen una voluntad de adaptación más estrecha a las diferentes necesidades de unos colectivos reconocidos como específicos. Muestra la tendencia a reducir la inserción a la sola cuestión del empleo.

\section{La inserción de los jóvenes en los años 1990}

El desempleo de los jóvenes está vinculado a las dificultades de inserción en el mercado laboral que se caracteriza por una sucesión de empleos precarios en el marco de la interinidad o de los dispositivos subvencionados. El aumento del número de jóvenes diplomados en búsqueda de empleo y el alargamiento de la duración necesaria para el acceso a un primer empleo no deben hacer olvidar el vínculo de causalidad existente entre el bajo nivel de cualificación y la dificultad de inserción profesional. Además, la concentración en la inserción económica no puede ocultar unas necesidades más amplias a propósito de las dimensiones sanitarias o las que se refieren a la vivienda. Ciertos jóvenes en gran dificultad social están lejos de poseer los recursos necesarios para asegurarse una inserción profesional. 
El equilibrio entre la prioridad dada al empleo y las competencias sociales necesarias debe buscarse en la individualización de las trayectorias y en la utilización de los recursos nacionales. Por último, los dispositivos existentes no tienen suficientemente en cuenta las especificidades de los jóvenes que viven en zonas rurales. La movilidad, la dinámica económica local y la adecuación de las cualificaciones a la economía local son unas cuestiones fundamentales que exigen unas respuestas adecuadas. La inserción de los jóvenes rurales se convierte en un aspecto de las políticas de desarrollo rural así como la de los jóvenes urbanos que ha sido integrada poco a poco en las políticas urbanas.

A lo largo de los años, el número de jóvenes que se presentan en las misiones locales y los PAIO aumenta hasta representar el 20\% de los que tienen entre 16 y 25 años en 1998, es decir 1,3 millones de jóvenes. El número de jóvenes diplomados se incrementa de manera importante. Este nivel de demanda, que no era imaginado cuando se crearon las misiones locales en 1982, provoca un atasco del dispositivo y una cierta urgencia mientras que, paralelamente, la crisis del empleo se consolida y cuestiona la idea de la formación como puerta de entrada eficaz para el empleo. Este doble fenómeno genera una interrogación básica sobre la capacidad de las misiones locales a hacer frente a la diversidad y a la amplitud de las demandas y sobre su vocación prioritaria a favor de los jóvenes en dificultad.

El Crédito formación individualizado $(\mathrm{CFI})^{9}$, el dispositivo PAQUE, o, más recientemente, el programa TRACE (Trayecto de acceso al empleo) apoyan estas misiones. No obstante, la acumulación de dispositivos complejiza a la vez la comprensión de las acciones de las Misiones locales y su financiación. Se trata de encontrar un equilibrio entre la puesta en marcha de dis-

${ }^{9} \mathrm{El} \mathrm{CFI}$, creado en septiembre de 1989, pretende favorecer el acceso a una cualificación reconocida de todos los jóvenes que han abandonado el sistema educativo sin ningún título académico, ofreciendo una segunda oportunidad a os jóvenes en dificultad de inserción. La ley quinquenal relativa al trabajo, los jóvenes en difcultad de inserción. La ley quinquenal relativa al trabajo, al empleo y a la formación profesional del 20 de diciembre de 1993, concede a la Región la plena competencia en materia de formación profesional continua de los jóvenes de menos de 26 años. La aplicación progresiva de la ley de descentralización político-administrativa instaura un periodo transitorio caracterizado por una competencia compartida entre el Estado y las regiones. positivos nacionales y la colaboración local (especialmente la implicación de los cargos electos), de luchar contra las disparidades financieras, de asegurar una mejor implantación local y de garantizar la puesta en red de los actores.

En 1997, se pone en marcha un nuevo dispositivo: el programa "nuevos servicios, nuevos empleos". Tiene como objetivo favorecer la creación de 350000 empleos, fomentando la creación de actividades y la aparición de nuevos oficios. Pretende romper con las lógicas existentes que no ofrecen, ni un verdadero empleo, ni un buen salario. En 1998, el dispositivo TRACE instaurado por la ley de lucha contra las exclusiones, se dirige a los jóvenes de menos de 25 años en gran dificultad. Está fundamentado sobre un acompañamiento personalizado, que permite una mejor toma en consideración de la situación individual y una búsqueda más eficaz de las respuestas. No contempla a priori unas medidas sino que se fundamenta sobre la idea de trayectoria para la cual moviliza unos medios en términos de formación y de perspectiva de empleo.

En 1997, Edith Cresson, comisaria europea, crea las escuelas de la segunda oportunidad cuya idea figura en el Libro blanco de la Comisión europea sobre la educación y la formación (1995) que fomenta igualmente el servicio voluntario de acción humanitaria. Los objetivos asignados a las escuelas de la segunda oportunidad son los siguientes:

- Reinsertar los jóvenes sin títulos académicos que provienen de los barrios desfavorecidos de las grandes concentraciones urbanas, en el marco de unos dispositivos que ofrecen una segunda oportunidad educativa.

- Asegurar o reforzar un encuadramiento notable por la contratación de profesores cualificados.

- Desarrollar entre los jóvenes afectados, la motivación, las capacidades de aprender a aprender, los conocimientos básicos y las habilidades sociales.

- Poner en marcha unas clases con un número reducido de alumnos.

Estas escuelas de la segunda oportunidad se inscriben en un movimiento más amplio de formación a lo largo de la vida. En 1999, el programa "Nuevas oportunidades" quiere reducir el número de jóvenes que salen del sistema educativo sin ninguna cualificación. 


\section{La ley de lucha contra las exclusiones}

La ley de lucha contra las exclusiones del 29 de julio de 1998 reorganiza profundamente las estructuras de inserción a través de la economía. Contempla, entre otros aspectos, la creación de unas obras de inserción para las personas que se encuentran muy alejadas del empleo y cuestiona las puestas a disposición de las empresas organizadas de las asociaciones intermedias. Según el artículo L.322 del Código del trabajo, modificado por la ley del 29 de julio de 1998: "la inserción por la actividad económica tiene como objeto permitir a unas personas sin empleo y que encuentran dificultades sociales y profesionales especiales, beneficiarse de los contratos laborales con el objetivo de facilitar su inserción social y profesional. El Estado puede, tras consultar los agentes locales reunidos en el seno del Consejo Departamental de la inserción a través de la actividad económica, firmar unos convenios con los empleadores. Estos convenios pueden contemplar unas ayudas estatales".

Cierto número de empresas de inserción se han posicionado en los sectores económicos dinámicos. Entre dinamismo económico y eficacia social, se sitúan en el mismo equilibrio delicado que los centros de trabajo protegido que acogen a unas personas en situación de dificultad. Esta tensión es aún mayor sabiendo que las financiaciones que reciben son poco duraderas, puesto que dependen de los planes gubernamentales que se suceden uno tras otro, provocando regularmente el cierre de ciertas estructuras. En los años 2000, surge una interrogación sobre el carácter a veces ilusorio de la inserción por la actividad económica. Según la FNARS ${ }^{10}$ (Federación Nacional de Acogida y de Reinserción Social) ${ }^{11}$, "en lugar de diseñar unas trayectorias hacia el empleo, crea una zona gris del empleo subvencionado por la administración pública de la que las per-

10 Creada en 1956, la FNARS es una red generalista de lucha contra las exclusiones. Reúne a 750 asociaciones u organismos que gestionan 2200 centros y servicios sociales que acogen, alojan e insertan a 600000 personas cada año. La federación tiene una misión política y de servicios, representa a sus miembros, los asesora, los informa y pone en común sus experiencias.

11 FNARS, (2006), Les Exclus et les travailleurs pauvres. Livre Blanc, juin 2006 sonas salen difícilmente. La instalación en la precariedad se convierte en una realidad". Paralelamente, poniendo el énfasis sobre la vuelta rápida al empleo, las orientaciones actuales de las políticas públicas privilegian "la inserción rápida de las personas fácilmente insertables, en detrimento de aquellas que son todavía incapaces, para las cuales la trayectoria de inserción es necesariamente más larga". No obstante, la comparación con el número de beneficiarios de la inserción a través de la actividad económica indica que este sector tiene una verdadera utilidad social, representa un peso económico no desdeñable y cumple una función ciudadana. Así, constituyen unos instrumentos al servicio del desarrollo económico y del empleo así como unos actores del acompañamiento de los colectivos en dificultad.

\section{De la ley de cohesión social al Grenelle de la inserción}

El 18 de enero de 2005 es aprobada la ley de programación para la cohesión social, conocida como Ley Borloo. Articulándose en torno a tres pilares principales: el empleo, la vivienda y la igualdad de oportunidades, tiene como objetivo apoyar a las personas más alejadas del mercado laboral y levantar los obstáculos administrativos y jurídicos de acceso al empleo. A través de esta ley, el rol de la inserción por la actividad económica es reconocido y sus recursos son reforzados:

- Aumento del $35 \%$ del número de puestos de trabajo financiados en las empresas de inserción.

- Generalización de los dispositivos de acompañamiento de las asociaciones intermedias.

- Creación de una ayuda al acompañamiento socio-profesional en los talleres y en las obras de inserción.

- Duplicación de la dotación del Estado al Fondo departamental de inserción.

El plan de cohesión social, en su programa $n^{\circ} 6$ titulado "Desarrollar la economía solidaria", refuerza el sector de la inserción a través de la actividad económica y fortalece los medios concedidos. Permite un reconocimiento legal de los talleres y de las obras de inserción y consolida los medios que 
le son concedidos, especialmente a través de la creación de una ayuda al acompañamiento. Reconoce por decreto la ayuda al acompañamiento en las asociaciones intermedias que había sido creada a título experimental en 2002 por una circular. En 2007, es elaborado un nuevo instrumento para mejorar la lucha contra las salidas del sistema educativo sin ningún título y favorecer el empleo de los jóvenes y la igualdad de oportunidades. Para ello se crea el esquema nacional de orientación y de inserción profesional de los jóvenes, que se denomina "Para un nuevo pacto con la juventud", organizado en torno a cuatro ejes:

- Una orientación continua y personalizada para formar a todos los jóvenes: descubrimiento de los oficios a partir de colegio, entrevistas individualizadas de orientación, etc.

- Una orientación activa hacia la enseñanza superior que prepara al empleo: único expediente de candidatura para la enseñanza superior, profesionalización de los estudios universitarios, aproximación entre las universidades y las empresas.

- Una notable coordinación nacional, regional y local para la información, la orientación y la inserción: ampliación de los comités de coordinación regionales a la formación profesional para la puesta en marcha de las políticas de inserción y de orientación

- Una movilización de todos los actores.

Además, en 2005, la ANRU (Agencia Nacional para la Renovación Urbana $)^{12}$ ha aprobado una carta nacional de inserción

12 La ANRU, instaurada por la ley del 1 de agosto de 2003 para la ciudad y la renovación urbana realiza un esfuerzo nacional sin precedentes de transformación de los barrios frágiles clasificados en Zonas Urbanas Sensibles (ZUS) o que se enfrentan a las mismas transformaciones socioeconómicas. Ello se traduce por la mejora de los espacios urbanos, el desarrollo de los equipamientos públicos, la rehabilitación y la "residencialización" de las viviendas sociales destinadas al alquiler, la demolición de las viviendas en razón de su vetustez o para mejorar la organización urbana de las viviendas, el desarrollo de una nueva mejorar la organización unvan imp de las vivis Pas, el desarillo de una nueva oferta de viviendas. La ANRU implementa el Programa Nacional de Renovación Urbana aprobando unos proyectos globales que financia gracias a unos fondo públicos y privados. La Agencia aporta su apoyo financiero a las administraciones locales, a los centros públicos y a los organismos privados que llevan a cabo unas operaciones de renovación urbana. Para 2013, alrededor de 500 barrios repartidos en todo el territorio francés serán renovados, mejorando la vida diaria de cerca de 4 millones de habitantes. que retoma, generalizándolo, el principio de la clausula de inserción en los mercados públicos. Así, cualquier operación financiada por la ANRU debe constar de una cláusula de inserción que se refiere a "los compromisos de inserción equivalentes, como mínimo, al 5\% del número total de horas trabajadas en el marco de los trabajos de inversión y un objetivo de al menos $10 \%$ de contrataciones directas o indirectas afectados por el marco de la gestión urbana de proximidad y de la gestión de los equipamientos que son el objeto de ayudas de la ANRU reservadas a los habitantes de las zonas urbanas sensibles (ZUS) ${ }^{13}$ ". El comité interministerial de lucha contra las exclusiones ha decidido en mayo de 2006 poner en marcha un programa de acciones para desarrollar las clausulas sociales en los mercados públicos estatales. Es preciso subrayar que la realización de una guía, que informa los compradores públicos sobre las diferentes modalidades jurídicas, permite realizar la inserción en el marco de un mercado público y proteger jurídicamente la utilización de las clausulas sociales.

Después del Grenelle del medioambiente, se ha abierto en noviembre de 2007, bajo la presidencia de Martin Hirsch, Alto Comisario a las Solidaridades activas contra la pobreza, un Grenelle de la inserción que debía concluir, al término de seis meses de trabajo, con unas propuestas de mejora de las políticas de inserción. Perseguía tres objetivos:

- Reubicar la inserción en el centro de los debates de la sociedad, dando la palabra a los profesionales, a las asociaciones y a los beneficiarios, y movilizando a las administraciones locales, a las empresas y a los agentes sociales.

${ }^{13}$ Las ZUS son unos territorios Infra-urbanos definidos por los poderes públicos por zer el objetivo prioritario de la politica urbana en función de las consid raciones locales vinculadas a las dificultades que conocen los habitantes de estos territorios. La ley del 1 estos teritorios. La ley del 14 de noviembre de 1906 que contempla la aplicación del pacto de reactivación de la política urbana distingue tres niveles de intervención: las zonas urbanas sensibles (ZUS), las zonas de reactivación urbana (ZRU) y las zonas francas urbanas (ZFU). Los tres niveles de intervención, caracterizados por unos dispositivos de orden fiscal y social, pretenden responder a unos niveles diferentes a las dificultades que se plantean en estos barrios. 
- Mejorar la eficacia global de las políticas de inserción.

- Emprender unas reformas que se impongan para llegar a una unificación de todos los contratos subvencionados por la administración pública con el fin de poner en marcha un contrato único de inserción; al acceso a la formación profesional para las personas más alejadas del empleo; y a la evolución de la financiación de la inserción a través de la actividad económica.

Sobre este último punto, Martin Hirsh se ha comprometido a que la inserción sea objeto de evaluaciones, en el seno de las empresas de inserción así como en las empresas clásicas que deberán "construir unos criterios de eficacia" en este ámbito. Está igualmente en proceso de estudio la puesta en marcha de una cláusula de inserción para las empresas. Tres grupos de reflexión trabajan sobre los siguientes temas: 1) los objetivos de la política de inserción y su gobernanza, 2) las trayectorias de inserción, y 3) el rol de los empleadores y su implicación en las políticas de inserción. Conviene subrayar que la ministra de Economía, Christine Lagarde, ha anunciado en 2008 que el proceso de modernización del marco de las EIAE (Estructura de Inserción por la Actividad Económica) debía conducir a la aprobación de nuevas reglas y, especialmente, a una concertación basada en una lógica del resultado, con unos objetivos negociados con las estructuras y unos indicadores compartidos que permitan evaluar los resultados obtenidos.

Entre los demás temas abordados durante los encuentros de la experimentación social, que tuvieron lugar en Grenoble en noviembre de 2008 para lanzar los trabajos del Grenelle de la inserción, se encuentran la reforma de los mínimos sociales, el microcrédito o el analfabetismo.

\section{Dispositivos elaborados y acciones implementadas}

Los poderes públicos han elaborado varios dispositivos y puesto en marcha múltiples acciones para favorecer la inserción a través de la actividad económica, entre los cuales se encuentran los fondos departamentales de inserción, las estructuras de inserción por la actividad económica, las clausulas sociales y la política de empleo de los jóvenes.

\section{Los fondos departamentales de inserción}

Los fondos departamentales de inserción (FDI) ${ }^{14}$ contribuyen al desarrollo del sector de la inserción a través de la actividad económica y deben permitir la realización de los objetivos fijados por el plan de cohesión social, especialmente a través de la creación de nuevas empresas de inserción, y las condiciones de desarrollo económico de estas estructuras. Los FDI deben favorecer la aproximación de las estructuras de inserción a través de la actividad económica y facilitar la evolución de las modalidades de organización de las estructuras del sector no mercantil. Los créditos de los fondos departamentales de inserción figuran en el presupuesto del ministerio del Empleo.

\section{Las estructuras de inserción por la actividad económica}

Creadas en el inicio de los años 1980, las estructuras de inserción a través de la actividad económica son uno de los principales instrumentos de lucha contra la enclusión. Acogen a más de un millón de personas cada año y su lema es: "Nadie es incontratable".

Las asociaciones intermedias: ponen el personal a la disposición de las empresas, de las asociaciones, de las administraciones locales o de los ciudadanos. Cuando la puesta a disposición es realizada para una empresa, es limitada a 240 horas. Las asociaciones intermedias aseguran la acogida y el seguimiento de las personas en gran dificultad en su proceso de inserción. Concertadas con el Estado, se benefician de unas reducciones de las cotizaciones sociales y de una ayuda al acompañamiento. Una de las especificidades de las asociaciones intermedias resulta de su función de acogida de estos públicos, anteriormente acogidos por otras estructuras de inserción, y están consideradas como unos referentes. Por lo tanto, el acompañamiento de los trabajadores contratados constituye la

${ }^{14}$ El FDI es una ayuda puntual concedida por el Consejo general para ayuda a los beneficiarios de la RMI, de la API y de la ASS, con el fin de favorecer la inserción social o profesional o de responder a unas dificultades vinculadas a una situación financiera precaria que pone en peligro el proceso de inserción. 
definición misma de las asociaciones intermedias. Desde 1998 su actividad ha dejado de limitarse "a la satisfacción de las necesidades colectivas no satisfechas". Han intervenido así en todos los sectores de actividad, sabiendo que una mayoría de las asociaciones intermedias interviene en el marco de la ayuda a domicilio. Aseguran, especialmente en zonas rurales, una acción a favor del mantenimiento de los vínculos sociales, de las personas mayores o de las familias. Cerca de 950 asociaciones intermedias emplean a cerca de 168000 personas.

Las empresas de inserción por la economía: unidades de producción de bienes mercantiles, ayudan, a través de la actividad productiva, a las personas en dificultad de reinserción en el mundo laboral. Concertadas con el Estado, reciben una ayuda para cada puesto de trabajo. Como verdaderas empresas, deben, a través de una actividad de producción y de contratos de inserción, favorecer la reinserción laboral de las personas acogidas así como asegurar su viabilidad económica. Han desarrollado unas actividades principalmente en la construcción y en los espacios verdes. Reciben unas ayudas del Estado destinadas a compensar los costes adicionales vinculados a la población acogida: rotación de la mano de obra, productividad reducida y acompañamiento social. Las personas están empleadas a través de los contratos temporales de una duración máxima de 24 meses. Cerca de 860 empresas de inserción emplean a 30400 personas.

Las empresas de trabajo temporal de inserción: concertadas con el Estado, funcionan como unas empresas de trabajo temporal. Utilizan las ofertas de empleo de un sector de actividad temporal para dar a las personas que se encuentran en gran dificultad la oportunidad de conseguir una experiencia profesional gratificante o una cualificación. Deben asegurar un seguimiento de las personas en dificultad de cara a favorecer su reinserción y reciben una subvención del Estado para el encuadramiento de estas personas. Cerca de 260 empresas de trabajo temporal de inserción emplean a 38000 personas.

Los talleres y obras de inserción: se dirigen a unas personas en situación de gran exclusión social. Funcionan sobre la base de contratos de trabajo de 6 a 24 meses, en el marco de un Contrato de futuro o de un Contrato de acompañamiento al empleo. Asocian la enseñanza y la producción, por una puesta en situa- ción de trabajo sobre verdaderas obras y pueden ser organizados puntualmente o de manera permanente. Desarrollan unas actividades de utilidad social que no son rentables, ni en las condiciones del derecho ordinario, ni en el marco de una empresa de inserción, en razón de las dificultades de organización que les son propias, especialmente la productividad limitada de los trabajadores contratados. Organizan "el seguimiento, el acompañamiento, el encuadramiento técnico y la formación de sus trabajadores de cara a facilitar su inserción social y a buscar las condiciones de una inserción profesional duradera". ${ }^{15}$ En 2006, la tasa de acceso y de vuelta al empleo de los trabajadores en inserción al término de su trayectoria debe alcanzar un mínimo del $20 \%$. Alrededor de 1600 talleres y obras de inserción emplean a 65000 personas.

Las corporaciones públicas de barrio: contratan sus trabajadores entre los habitantes del barrio. Aseguran un mantenimiento de los espacios verdes, la limpieza, el mantenimiento técnico y participan en las operaciones de rehabilitación de edificios. La necesidad de un equilibrio económico impone una serie de dificultades vinculadas al tamaño insuficiente del barrio concernido para generar un volumen de negocios suficiente. Pueden ser concertadas como empresas de inserción y las cerca de 130 corporaciones públicas de barrio emplean a 10000 personas.

Las cláusulas sociales

La mayoría de las estructuras de inserción se implican en nuevos segmentos, poco o mal ocupados por la economía tradicional, de modo que esta última se ha dirigido a veces a este sector acusándolo de competencia desleal. Desde 1993, unas cláusulas sociales pueden influir sobre la concesión de contratos públicos. No obstante, esta cláusula social ha sido considerada ilegal por una decisión del tribunal de Estrasburgo dictaminada en 1999.

Este instrumento todavía poco utilizado tiende a desarrollarse bajo la influencia de la ANRU que contempla unos com- 
promisos de inserción para todas las acciones de inversión o de funcionamiento que financia. La puesta en marcha de esta cláusula sigue siendo difícil y exige una voluntad y una cooperación importante de los actores, tanto de las empresas como de las estructuras de inserción. Así, el 5\% de las horas de inserción previstas por las cláusulas sociales aparece, como insuficiente para crear las condiciones de una inserción duradera para las personas concernidas. Por lo tanto, ciertas empresas se agrupan para responder a las obligaciones y dan un sentido a las iniciativas de inserción. Deben hacer frente a numerosos obstáculos y especialmente a la difícil adecuación entre las necesidades de competencia de las empresas y la falta de formación de un gran número de personas en búsqueda de empleo. Se construyen unas pasarelas en torno al camino de formación, de cualificación y de acceso al empleo que abre unas perspectivas interesantes para los actores de la inserción a través de la economía.

\section{La política de empleo de los jóvenes}

Se sustenta en tres dispositivos: 1) un dispositivo de acogida, de información y de orientación, 2) un dispositivo de formación y de inserción profesional, y 3 ) un dispositivo de contratos subvencionados.

Las Misiones locales y las PAIO constituyen las bases del primer dispositivo. Gestionan además el dispositivo de contratos subvencionados por la administración pública en relación con los servicios del Estado. Las Misiones locales, creadas después del informe Schwartz de 1981 sobre la inserción social y profesional de los jóvenes en dificultad, son hoy en día cerca de 420. Trabajan en relación con las 72 PAIO (Permanencias de acogida, de información y de orientación) y ponen en marcha unas acciones orientadas principalmente hacia el empleo y la formación. En 2006, las Misiones locales y las PAIO han acogido por primera vez a 477000 jóvenes y han estado en contacto con más de 1200000 jóvenes, empleando a más de 10000 trabajadores.

Los comités locales para el alojamiento autónomo de los jóvenes: creados en 1980, aseguran una misión de acogida, de información y de orientación de los jóvenes en búsqueda de alo- jamiento. Algunas gestionan igualmente unos parques inmobiliarios que permiten así evitar el problema de la escasa confianza de los arrendadores hacia los jóvenes en situación precaria.

Los puntos de acogida o los puntos de escucha: son creados 70 puntos de escucha en 1996 y otros se ponen en marcha bajo nuevos estatus. Comparten el hecho de ofrecer unos vínculos abiertos, con un bajo nivel de exigencia, en donde la escucha prima sobre la acción y la palabra sobre la elaboración de un proyecto.

La formación y la inserción profesional dependen, en términos de misiones, a la vez del ministerio de Educación, de la DDTEFP ${ }^{16}$ y de los Consejos generales. Están puestas en marcha por unos actores múltiples, unos organismos de formación y unos servicios de acompañamiento social y profesional. Unos programas anuales definen las orientaciones en términos de colectivos y de campo de formación y constituyen unos pliegos de condiciones en los cuales se inscriben los organismos de formación y de inserción profesional. En este sentido, la inserción social y profesional ha dejado de ser un asunto de especialistas. Es tomada en consideración por los actores de las políticas de juventud, movimientos de educación popular, equipos de prevención especializados o centros educativos, que multiplican las acciones y las experimentaciones. Alternativamente integrados en la puesta en marcha de los nuevos dispositivos o creadores de nuevas acciones, participan a la movilización de toda la sociedad.

\section{Conclusión}

Recordemos que, a lo largo de los últimos treinta años, Francia ha favorecido el desarrollo de un derecho a la inserción, especialmente vía la Renta Mínima de Inserción (RMI) y las estructuras de inserción por la economía, pasando así de un derecho a la asistencia a un derecho a la inserción. Esta política se ha traducido a la vez por la creación de estructuras (empre-

${ }^{16}$ Dirección Departamental del Trabajo, del Empleo y de la Formación Profesional. 
sas de inserción, asociaciones de inserción, agrupaciones de empleadores para la inserción y formación) y unas medidas individuales sinónimas de contratos subvencionados por la administración pública (Contratos Empleo Solidaridad, Contratos Iniciativas Empleo, Empleos Jóvenes, Contratos Empleo Consolidado). La inserción a través de la economía, que se ha convertido en la inserción por la actividad económica, es uno de los apartados de la economía social y solidaria. Reúne a un conjunto de estructuras y de dispositivos destinados a favorecer la reinserción profesional de unos públicos fragilizados o marginados para los cuales las perspectivas de acceso al empleo suponen unas condiciones previas en materia de salud y de habilidades sociales.

\section{Bibliografía}

ABHERVÉ, M. y LABBÉ, M. (2005). L'insertion professionnelle et sociale des jeunes ou l'intelligence pratique des missions locales. Rennes: Editions Apogée.

BELORGEY, J-M. (2000). Minima sociaux, revenus d'activité, précarité. Paris: La Documentation française.

CASTRA, D. (2003). L'insertion professionnelle des publics précaires. Paris: PUF.

CHARIOT, B. y GLASSMAN, D. (1998). Les Jeunes, l'insertion et l'emploi. Paris: PUF.

DRESS, (2007). "Les politiques d'insertion des conseils généraux en direction des bénéficiaires du RMI", Etudes et Résultats, $\mathrm{n}^{\circ} 582$, juillet, 2007.

FNARS, (2006). Les exclus de l'emploi et les travailleurs pauvres. Livre blanc, juin 2006.

GUÉRIN-PLANTIN, C. Genèses de l'insertion, l'action publique indéfinie. Paris: Dunod.

PRATIQUES, (2007). "L'insertion au service de l'emploi, Alternatives économiques", $\mathrm{n}^{\circ}$ 30, septembre 2007.

SCHWARTZ, B. (2007). Rapport sur l'insertion professionnelle et sociale des jeunes. Rennes: Editions Apogée.

ROSE, J. (1998). Les jeunes face à l'emploi. Paris: Desclée de Brouwer.

THÉVENET, A. (1994). RMI, théorie et pratique. Paris: Bayard. 